\title{
Diagnostic value of levels of presepsin (soluble CD14-subtype) in febrile neutropenia in patients with hematological disorders
}

Hideo Koh, Mizuki Aimoto, Takako Katayama, Masamichi Hashiba, Ayumi Sato, Masatomo Kuno, Yosuke Makuuchi, Teruto Takakuwa, Hiroshi Okamura, Asao Hirose, Mika Nakamae, Masayuki Hino, Hirohisa Nakamae

\begin{tabular}{|c|l|}
\hline Citation & Journal of Infection and Chemotherapy, 22(7); 466-471 \\
\hline Issue Date & 2016 -07 \\
\hline Type & Journal Article \\
\hline Textversion & Author \\
\hline \multirow{2}{*}{ Rights } & $\begin{array}{l}\text { O2016 Japanese Society of Chemotherapy and The Japanese Association for Infectious } \\
\text { Diseases. This manuscript version is made available under the CC-BY-NC-ND } 4.0 \\
\text { License. http://creativecommons.org/licenses/by-nc-nd/4.0/ } \\
\text { This is the accept manuscript version. The article has been published in final form at } \\
\text { https://doi.org/10.1016/j.jiac.2016.04.002 }\end{array}$ \\
\hline DOI & \begin{tabular}{l}
$10.1016 / j . j i a c .2016 .04 .002$ \\
\hline
\end{tabular}
\end{tabular}

Self-Archiving by Author(s)

Placed on: Osaka City University

$\mathrm{KOH} \mathrm{H}$, et al. (2016). Diagnostic value of levels of presepsin (soluble CD14-subtype) in febrile neutropenia in patients with hematological disorders. Journal of Infection and Chemotherapy: Official Journal of the Japan Society of Chemotherapy. 22, 466-471. 
Manuscript Number: JIC-D-16-00062R1

Title: Diagnostic value of levels of presepsin (soluble CD14-subtype) in febrile neutropenia in patients with hematological disorders

Article Type: Original Article

Section/Category: HIV / AIDS / Opportunistic Infection / Hepatitis

Keywords: presepsin (soluble CD14-subtype); febrile neutropenia; procalcitonin; C-reactive protein; interleukin-6; and interleukin-8

Corresponding Author: Dr Hideo Koh, Ph.D., M.D.

Corresponding Author's Institution:

First Author: Hideo Koh, Ph.D., M.D.

Order of Authors: Hideo Koh, Ph.D., M.D.; Mizuki Aimoto; Takako Katayama; Masamichi Hashiba; Ayumi Sato; Masatomo Kuno; Yosuke Makuuchi; Teruto Takakuwa; Hiroshi Okamura; Asao Hirose; Mika Nakamae; Masayuki Hino; Hirohisa Nakamae

Abstract: Background: Whether presepsin (soluble CD14-subtype) is better than other markers including procalcitonin (PCT), has not been adequately investigated in febrile neutropenia (FN) .

Methods: We prospectively examined the utility of presepsin in FN in Cohort 1 (C1) and 2 (C2), between November 2010 and February 2012, and between November 2013 and January 2014, respectively. The purpose of this study was to investigate 1 ) the relative value of serum presepsin over serum $\mathrm{PCT}$ in $\mathrm{Cl}$, and 2) the relative value of plasma presepsin as compared with serum PCT, C-reactive protein, interleukin-6 and interleukin-8 with frequent, repeated sampling in $\mathrm{C} 2$.

Results: Seventy-nine FN episodes $(\mathrm{C} 1,75 ; \mathrm{C} 2,4)$ were evaluable. In $\mathrm{C} 1$, when compared with control values, presepsin was significantly higher at onset of $\mathrm{FN}(\mathrm{P}=0.004)$, while $\mathrm{PCT}$ was not significantly higher $(\mathrm{P}=$ 0.54 ). The median value of serum presepsin within 72 hours of onset of $F N$ in subjects with fever of unknown origin, local infection, bacteremia and septic shock was 680 (reference 314) pg/ml, 763, 782 and 1359, respectively. In $\mathrm{C} 2$, the mean levels of plasma presepsin from onset of $\mathrm{FN}$ to 72 hours were classified as negative in the two patients with no suspected site of infection, and those of the remaining two patients with clinically probable infections were positive (175, 131, 346 and 329 $\mathrm{pg} / \mathrm{ml}$, respectively). In contrast, the other markers did not discriminate between this two groups.

Conclusions: In FN, presepsin may be an earlier and more sensitive indicator of bacterial infection than PCT. 


\section{ORIGINAL ARTICLE}

Diagnostic value of levels of presepsin (soluble CD14-subtype) in febrile neutropenia in patients with hematological disorders

Authors: Hideo Koh ${ }^{1}$, M.D., Ph.D., Mizuki Aimoto ${ }^{1}$, M.D., Ph.D., Takako Katayama ${ }^{1}$, Masamichi Hashiba ${ }^{2}$, Ph.D., Ayumi Sato ${ }^{2}$, MSc., Masatomo Kuno ${ }^{1}$, M.D., Yosuke Makuuchi $^{1}$, M.D., Teruto Takakuwa ${ }^{1}$, M.D., Hiroshi Okamura ${ }^{1}$, M.D., Asao Hirose ${ }^{1}$, M.D., Ph.D., Mika Nakamae ${ }^{1}$, M.D., Ph.D., Masayuki Hino ${ }^{1}$, M.D., Ph.D., and Hirohisa Nakamae ${ }^{1}$, M.D., Ph.D.

Institutions: ${ }^{1}$ Hematology, Graduate School of Medicine, Osaka City University, Osaka, Japan

${ }^{2}$ Development Research, Mochida Pharmaceutical Co., Ltd., Shizuoka, Japan

\section{Source of Funding}

This work was funded by Mochida Pharmaceutical Co., Ltd.

Correspondence: Hideo Koh, M.D., Ph.D.

Hematology, Graduate School of Medicine, Osaka City University,

1-4-3, Asahi-machi, Abeno-ku, Osaka 545-8585, Japan 
Phone: +81-6-6645-3881, Fax: +81-6-6646-3880

E-mail: hidekoh@med.osaka-cu.ac.jp

RUNNING TITLE: Presepsin (soluble CD14-subtype) for febrile neutropenia

\author{
Abstract: 248 words, Text: 2586 words
}




\begin{abstract}
Background: Whether presepsin (soluble CD14-subtype) is better than other markers including procalcitonin (PCT), has not been adequately investigated in febrile neutropenia $(\mathrm{FN})$.

Methods: We prospectively examined the utility of presepsin in FN in Cohort 1 (C1) and 2 (C2), between November 2010 and February 2012, and between November 2013 and January 2014, respectively. The purpose of this study was to investigate 1) the relative value of serum presepsin over serum PCT in $\mathrm{C} 1$, and 2) the relative value of plasma presepsin as compared with serum PCT, C-reactive protein, interleukin-6 and interleukin- 8 with frequent, repeated sampling in $\mathrm{C} 2$.
\end{abstract}

Results: Seventy-nine FN episodes $(\mathrm{C} 1,75 ; \mathrm{C} 2,4)$ were evaluable. In $\mathrm{C} 1$, when compared with control values, presepsin was significantly higher at onset of FN $(P=0.004)$, while PCT was not significantly higher $(P=0.54)$. The median value of serum presepsin within 72 hours of onset of FN in subjects with fever of unknown origin, local infection, bacteremia and septic shock was 680 (reference 314) pg/ml, 763, 782 and 1359, respectively. In C2, the mean levels of plasma presepsin from onset of FN to 72 hours were classified as negative in the two patients with no suspected site of infection, and those of the remaining two patients with clinically probable infections were positive $(175,131,346$ and $329 \mathrm{pg} / \mathrm{ml}$, respectively). In contrast, the other markers did not discriminate between this two groups. 
Conclusions: In FN, presepsin may be an earlier and more sensitive indicator of bacterial infection than PCT.

Key words: presepsin (soluble CD14-subtype), febrile neutropenia, procalcitonin, C-reactive protein, interleukin-6, and interleukin-8 


\section{Introduction}

Febrile neutropenia (FN) remains one of the major complications during chemotherapy in patients with solid tumors or hematological malignancies, despite recent progress in strategies that prevent infection. As blood cultures can identify infectious etiologies of FN with a likelihood of just $20 \%$ [1], a fever-driven approach using broad-spectrum antibiotics is still common in daily practice, probably resulting in overtreatment, increased costs, and induction of drug-resistance in bacteria. Previous reports showed that procalcitonin (PCT), C-reactive protein (CRP) and some cytokines such as interleukin (IL)-6 and IL-8 may be helpful in diagnosing or ruling out bacterial infections in the management of FN [2-11]. To date, no strategy based on these tests has been established as a standard of care for FN.

In 2005, presepsin (soluble CD14-subtype) was reported to be a novel, superior diagnostic biomarker of infection, especially of sepsis, compared with PCT or IL-6 [12], and several recent prospective studies in the field of critical care medicine have reported its usefulness for diagnosis of sepsis or prediction of mortality [13-15]. Presepsin was identified as a $13 \mathrm{kD}$ molecule comprising the soluble fraction of $\mathrm{CD} 14$, one of the surface markers in monocytes/macrophages that binds the lipopolysaccharide (LPS)-LPS binding protein $[16,17]$. Although the exact mechanism of its production is not completely understood, monocytes may secrete it in humans, triggered by bacterial phagocytosis or a sterile phagocytic stimulus [18]. Only one recent prospective study investigated the diagnostic value of presepsin in 
pediatric oncology patients with FN [19]. Urbonas et al. showed that plasma levels of presepsin did not provide diagnostic power to differentiate fever of unknown origin (FUO) from bacteremia/sepsis, while PCT might be used as an additional diagnostic tool for detecting bacteremia/sepsis [19]. However, there is a paucity of data on which to conclude whether or not presepsin is better than other markers including PCT.

On the basis of these observations, we prospectively examined the utility of presepsin levels in adult patients with hematological disorders and FN. The purpose of this study was to evaluate whether presepsin was a better diagnostic marker for bacterial infections in comparison with PCT, CRP, IL-6 and IL-8.

\section{Patients and methods}

\section{Study design}

We conducted a prospective, observational study consisting of two cohorts (Cohort 1 and Cohort 2) to examine the utility of presepsin levels for detecting bacterial infections in $\mathrm{FN}$, compared with previously reported markers. We aimed to investigate whether presepsin is more useful than procalcitonin in Cohort 1, and assess the superiority of presepsin as compared with PCT, CRP, IL-6 and IL-8 in Cohort 2.

In Cohort 1, we used serum samples and data collected prospectively between November 2010 and February 2012, according to the protocol for prospective cohort study of FN at our 
institution [20]. Since sampling points we designed were before chemotherapy (control data), at onset of FN and within 72 hours of FN in Cohort 1, we did not closely monitor the kinetics of biomarkers in FN. In Cohort 2, we therefore planned to institute close monitoring in a limited number of patients with FN (four), and blood samples were collected between November 2013 and January 2014. This study was approved by the Human Subjects Review Committee at Osaka City University and signed, informed consent was obtained from all patients.

\section{Study subjects and sample collection}

Inclusion criteria in this study were as follows: patients aged 16 years or older with hematological disorders; patients who received chemotherapy including conditioning regimens for hematopoietic cell transplantation; and patients who developed new-onset FN. FN was diagnosed if a patient had an axillary temperature of $\geq 37.5^{\circ} \mathrm{C}$ at a single measurement with a neutrophil count $<500 / \mu 1$ or $<1,000 / \mu 1$ with an expected decline to $<500 / \mu 1$, with reference to previously reported criteria $[21,22]$. Methods for collecting and storing serum samples in Cohort 1 have been described previously [20]. Serum and plasma samples in Cohort 2 were collected at the following times: once before chemotherapy (control); three times per week until onset of FN after chemotherapy; at onset of FN, at 8, 16, 24, 48 and 72 hours after the onset of FN; and every 48 hours thereafter until resolution of 
fever, in accordance with the protocol.

\section{Clinical assessment and definitions}

At onset of FN, as a rule, two sets of blood cultures were taken: one from venipuncture and another set from a central venous device if the patient had one. The following assessments were performed: physical and blood examination; cultures of samples from suspected sites of infection; and imaging tests including computed tomography.

We defined the four classifications described below, modifying the criteria so that they were applicable in patients with FN [23,24]. In addition, we also defined drug fever. Categorization of FN events was reviewed by two independent investigators and a final diagnosis was made. FUO was diagnosed if a patient had no signs or symptoms of infection except fever. Local infection was diagnosed if a patient had focal signs or symptoms of infection, regardless of microbiological evidence. Bacteremia was diagnosed if a patient had a positive blood culture; bacteremia due to coagulase negative staphylococcus (CNS) was diagnosed if we obtained at least two isolations of CNS with identical antibiograms, taken from different sites; otherwise the result was regarded as contamination [4]. Septic shock was diagnosed if a patient met the criteria for sepsis, apart from the white blood cell count, and if they presented with hypotension despite adequate fluid resuscitation, or the patient was dependent on inotropic or vasopressor agents. Drug fever was diagnosed if a patient met all 
of the following criteria: origin of fever was unknown; the febrile response or defervescence accorded with the timing of drug use; and the drug was one of the well-known drugs that often cause fever [25].

\section{Measurements of presepsin and other biomarkers}

In Cohort 1, serum levels of presepsin were determined by a two-step, enzyme-linked immunosorbent assay using recombinant sCD14-ST as the standard [12]. In Cohort 2, plasma levels of presepsin were measured by the PATHFAST ${ }^{\circledR}$ Presepsin kit (LSI Medience Corporation, Japan) [26]. Serum levels of PCT, CRP and cytokines were measured by electrochemiluminescence immunoassay, nephelometry and the Bio-Plex Pro Cytokine Assay ${ }^{\circledR}$ system (Bio-Rad Laboratories, CA) [27], respectively. A result was considered to be positive if it met the following criteria: $314 \mathrm{pg} / \mathrm{ml}$ for presepsin, $0.05 \mathrm{ng} / \mathrm{ml}$ for PCT, $0.30 \mathrm{mg} / \mathrm{dl}$ for CRP, $9.00 \mathrm{pg} / \mathrm{ml}$ for IL-6 and $116.00 \mathrm{pg} / \mathrm{ml}$ for IL-8; the cut-off value for detecting sepsis was $500 \mathrm{pg} / \mathrm{ml}$ for presepsin and $0.50 \mathrm{ng} / \mathrm{ml}$ for PCT, according to the respective manufacturer's instructions.

\section{Statistical analysis}

To compare two groups, we used paired or unpaired t-test for normally distributed variables; otherwise we performed the Wilcoxon signed-rank or Mann-Whitney test. To 
compare three or more groups, we used the Kruskal-Wallis test, and then employed the Steel test in post hoc analysis. A monotonic trend of variables across the groups was evaluated by the Jonckheere-Terpstra test. All $P$ values and $95 \%$ CIs were calculated using two-tailed tests. A $P$ value of less than 0.05 was deemed statistically significant. All statistical analyses were performed using IBM $^{\circledR}$ SPSS $^{\circledR}$ Statistics, version 22.0, Graph Pad Prism ${ }^{\circledR}$ version 5.02 (Graph Pad Software Inc., San Diego, CA, USA), and EZR, version 1.28, a modified version of $\mathrm{R}$ commander (version 2.1-7) based on the statistical software environment $\mathrm{R}$ (version 3.2.0) [28].

\section{Results}

\section{Patients}

A total of 47 patients were enrolled in cohorts 1 and 2, and a total of 79 FN episodes were evaluable. The details of patients' background and FN events are shown in Table 1, and the four patients in Cohort 2 were termed P1 (patient no.1), P2, P3 and P4. In Cohort 1, according to the protocol, 75 serum samples were collected within $72 \mathrm{~h}$ of onset of FN, 12 as controls before chemotherapy, and 24 at the onset of FN. In Cohort 2, the four patients (P1, P2, P3 and P4) were sampled 18, 17, 18 and 15 times, respectively.

\section{Cohort 1}


Of the 12 patients with samples both as a control and within 72 hours after the onset of $\mathrm{FN}$, the mean value of presepsin within 72 hours of FN (649 pg/ml, range 314-1134) was significantly higher than that in controls $(436,262-846)$ (paired-t $P<0.001$ ); the median value of PCT within 72 hours of FN $(0.12 \mathrm{ng} / \mathrm{ml}, 0.05-0.40)$ was also significantly higher than that of PCT as controls $(0.068 \mathrm{ng} / \mathrm{ml}, 0.02-0.14)$ (Wilcoxon signed-rank $P=0.002)$.

To investigate the influence of sampling time on the dynamics of presepsin and PCT, we compared serum levels of these two markers against sample collection time (Fig. 1). When compared with controls, presepsin was significantly higher at onset, from 0 to $24 \mathrm{~h}$ and from 24 to 48 h (Steel $P=0.004, P=0.002$ and $P=0.0003$, respectively). In contrast, when compared with controls, PCT was not significantly higher at onset, but significantly higher from 0 to $24 \mathrm{~h}$ and from 24 to $48 \mathrm{~h}$ (Steel $P=0.54, P=0.001$ and $P=0.009$, respectively).

We compared the levels of presepsin with those of PCT across the classification of causes of FN (Fig. 2). There was no significant difference with presepsin (Kruskal-Wallis $P=0.65$ ), whereas there was a significant difference with PCT (Kruskal-Wallis $P=0.02$ ). In contrast, there was a weak, monotonically increasing trend in presepsin $(P=0.089)$, while there was no significant trend with PCT $(P=0.105)$. In these statistical analyses, the "others" group was not included.

Of the 72 FN episodes except for the "others" group, we evaluated serum levels of presepsin and PCT that were measured within 72 hours after onset of FN, comparing the 
septic shock group $(n=5)$ and the other groups $(n=67)$. The mean value of presepsin in patients with septic shock (1170 pg/ml, 493-2115) was not significantly higher than that of presepsin in patients without septic shock $(851,314-2615)$ (unpaired-t $P=0.143$ ), whereas the median value of PCT in patients with septic shock $(3.58 \mathrm{ng} / \mathrm{ml}, 0.16-6.22)$ was significantly higher than that of PCT in patients without septic shock $(0.16 \mathrm{ng} / \mathrm{ml}, 0.03-5.80)$ (Mann-Whitney $P=0.002$ ).

To explore the potential of presepsin for detecting bacterial infections in FN, we evaluated the scatter plots of presepsin and PCT in patients with clinically documented infections, with reference to previously reported cut-off values of sepsis: $500 \mathrm{pg} / \mathrm{ml}$ for presepsin and $0.50 \mathrm{ng} / \mathrm{ml}$ for PCT (Fig. 3). Most of the plots of the patients diagnosed as local infection or bacteremia were distributed in the area with a presepsin level of $\geq 500 \mathrm{pg} / \mathrm{ml}$ and a procalcitonin level of $<0.50 \mathrm{ng} / \mathrm{ml}$ (Fig. 3a, 3b).

\section{Cohort 2}

A total of four patients aged 29-54 years were enrolled and four FN episodes were evaluable. The underlying diseases were acute leukemia in three patients (P1, P2, P3) and one non-Hodgkin's lymphoma (P4). Regarding the cause of FN, P1-P4 were finally diagnosed as FUO, suspected drug fever, clinically documented local infection and Staphylococcus aureus bacteremia, respectively. The patterns of fever and kinetics of plasma presepsin and serum 
biomarkers during FN episodes are shown in Fig. 4.

The presepsin peak levels from the onset of FN to 24 hours in P1, P2, P3 and P4 were 330, 148, 348 and $460 \mathrm{pg} / \mathrm{ml}$, respectively. The corresponding levels of PCT, CRP, IL-6 and IL-8 in $\mathrm{P} 1, \mathrm{P} 2, \mathrm{P} 3$ and $\mathrm{P} 4$ were $0.04,0.34,0.36$ and $0.22 \mathrm{ng} / \mathrm{ml} ; 1.8,6.0,11.9$ and $4.9 \mathrm{mg} / \mathrm{dl} ; 14$ (baseline value before chemotherapy, 27), 107 (23), 69 (25) and 24 (8.4) pg/ml; and 52 (62), 787 (58), 56 (150) and 26 (13) pg/ml, respectively. In P2, where no infection was suspected, presepsin was the only marker that did not increase.

The mean levels of presepsin from the onset of FN to 72 hours (6 measurements per patient) in P1, P2, P3 and P4 were 175 (95\%CI 93-257), 131 (108-153), 346 (289-404) and 329 (257-401) pg/ml, respectively. The corresponding levels of PCT, CRP, IL-6 and IL-8 in P1, P2, P3 and P4 were 0.02 (95\%CI 0-0.04), $0.16(0.01-0.31), 0.34$ (0.24-0.45) and 0.19 $(0.15-0.22) \mathrm{ng} / \mathrm{ml} ; 1.0(0.4-1.6), 3.1(0.9-5.3), 9.9(6.3-13.5)$ and $3.5(2.3-4.7) \mathrm{mg} / \mathrm{dl} ; 6.5$ (0.6-12), $23(0-66), 48(21-75)$ and $18(12-24) \mathrm{pg} / \mathrm{ml}$; and 38 (28-48), $160(0-483), 53$ (31-75) and $25(22-29) \mathrm{pg} / \mathrm{ml}$, respectively. For presepsin alone, the mean levels were positive in both $\mathrm{P} 3$ and $\mathrm{P} 4$, who most likely had a bacterial infection, and negative in both $\mathrm{P} 1$ and P2, who likely had no bacterial infection.

\section{Discussion}

In the present study, we demonstrated that in patients with $\mathrm{FN}$, presepsin levels were 
elevated significantly earlier than were PCT levels. In addition, we found that presepsin may be a more sensitive indicator of bacterial infection, although the ability of presepsin to discriminate septic shock from other conditions was inferior to that of PCT.

To the best of our knowledge, just one recent, prospective data set is available concerning the utility of presepsin in FN [19]. Urbonas et al. showed that there was no significant difference in plasma presepsin levels between their FUO (including local infection) and bacteremia/sepsis groups (median 356 pg/ml, 95\%CI 255-469; median 401, 95\%CI 212-484, respectively). In the present study, as in the report by Urbonas, we did not show a result that discriminated significantly between septic shock and other conditions (unpaired-t $P=0.143$ ). However, the median level of serum presepsin in the group with septic shock was clearly the highest when compared with other groups, despite not attaining significance (Fig. 2), and it is possible that an insufficient number of cases may have led to these statistically non-significant results. In addition, that negative result of Urbonas' data on presepsin [19] might be affected by the classification of causes of FN.

Although we did not employ statistical tests due to the small sample size, the results obtained from Cohort 2 were interesting. With reference to a plasma cut-off value of 314 $\mathrm{pg} / \mathrm{ml}$ for presepsin [26], only presepsin appeared to discriminate between FN with a low probability of bacterial infections ( $\mathrm{P} 1$ or $\mathrm{P} 2)$ and probable bacterial infections ( $\mathrm{P} 3$ or $\mathrm{P} 4)$. This suggests that presepsin might be more useful than CRP, PCT, IL-6 and IL-8 in the 
management of FN, especially when attempting to rule out the presence of serious bacterial infections. Therefore, it seems to be worth investigating the utility of presepsin for FN, especially in patients with the diagnosis of FUO.

Although the precise pathophysiology of elevated presepsin levels in patients with FN remains unclear, monocytes and elastase may contribute to this phenomenon [18]. Arai et al. showed that presepsin may be secreted from monocytes after phagocytosis of bacteria and elastase may cleave CD14 into presepsin in monocytes. From the results of the present study (Fig.1), we found that presepsin showed a clear increase in FN despite the decreased number of monocytes during chemotherapy.

The present study has several limitations. We did not perform close and frequent sampling after onset of FN in Cohort 1. In addition, sample size was not sufficient to draw definitive conclusions on comparison with other biomarkers. However, this study has some strengths. One was its prospective design and another was that the data for each biomarker at each point was measured at the same time in the same sample, leading to more objective comparisons.

Collectively, presepsin may respond to bacterial infections in a timely manner and be a more sensitive biomarker for these than PCT in FN. To establish the position of presepsin in management of FN, further future study is needed. 


\section{Conflict of interest}

H Nakamae received grants from Mochida Pharmaceutical Co., Ltd.

\section{Acknowledgment}

This work was funded by Mochida Pharmaceutical Co., Ltd. 


\section{References}

1. Klastersky J, Ameye L, Maertens J, Georgala A, Muanza F, Aoun M, et al. Bacteraemia in febrile neutropenic cancer patients. Int J Antimicrob Agents. 2007;30(Sup 1): S51-59.

2. Giamarellos-Bourboulis EJ, Grecka P, Poulakou G, Anargyrou K, Katsilambros N, Giamarellou H. Assessment of procalcitonin as a diagnostic marker of underlying infection in patients with febrile neutropenia. Clin Infect Dis. 2001;32:1718-25.

3. Jimeno A, García-Velasco A, del Val O, González-Billalabeitia E, Hernando S, Hernández $\mathrm{R}$, et al. Assessment of procalcitonin as a diagnostic and prognostic marker in patients with solid tumors and febrile neutropenia. Cancer. 2004;100:2462-9.

4. Giamarellou H, Giamarellos-Bourboulis EJ, Repoussis P, Galani L, Anagnostopoulos N, Grecka $\mathrm{P}$, et al. Potential use of procalcitonin as a diagnostic criterion in febrile neutropenia: experience from a multicentre study. Clin Microbiol Infect. 2004;10:628-33.

5. Manian FA. A prospective study of daily measurement of C-reactive protein in serum of adults with neutropenia. Clin Infect Dis. 1995;21:114-21.

6. Haeusler GM, Carlesse F, Phillips RS. An updated systematic review and meta-analysis of the predictive value of serum biomarkers in the assessment of fever during neutropenia in children with cancer. Pediatr Infect Dis J. 2013;32:e390-6.

7. Phillips RS, Wade R, Lehrnbecher T, Stewart LA, Sutton AJ. Systematic review and meta-analysis of the value of initial biomarkers in predicting adverse outcome in febrile 
neutropenic episodes in children and young people with cancer. BMC Med. 2012;10:6.

8. Urbonas V, Eidukaite A, Tamulienė I. The diagnostic value of interleukin-6 and interleukin- 8 for early prediction of bacteremia and sepsis in children with febrile neutropenia and cancer. J Pediatr Hematol Oncol. 2012;34:122-7.

9. Miedema KGE, de Bont ESJM, Elferink RFMO, van Vliet MJ, Nijhuis CSMO, Kamps WA, et al. The diagnostic value of CRP, IL-8, PCT, and sTREM-1 in the detection of bacterial infections in pediatric oncology patients with febrile neutropenia. Support Care Cancer. 2011;19:1593-600.

10. Kitanovski L, Jazbec J, Hojker S, Derganc M. Diagnostic accuracy of lipopolysaccharide-binding protein for predicting bacteremia/clinical sepsis in children with febrile neutropenia: comparison with interleukin-6, procalcitonin, and C-reactive protein. Support Care Cancer. 2014;22:269-77.

11. García de Guadiana-Romualdo L, Español-Morales I, Cerezuela-Fuentes P, Consuegra-Sánchez L, Hernando-Holgado A, Esteban-Torrella P, et al. Value of lipopolysaccharide binding protein as diagnostic marker of infection in adult cancer patients with febrile neutropenia: comparison with C-reactive protein, procalcitonin, and interleukin 6. Support Care Cancer. 2015;23:2175-82.

12. Yaegashi Y, Shirakawa K, Sato N, Suzuki Y, Kojika M, Imai S, et al. Evaluation of a newly identified soluble CD14 subtype as a marker for sepsis. J Infect Chemother. 
$2005 ; 11: 234-8$.

13. Endo S, Suzuki Y, Takahashi G, Shozushima T, Ishikura H, Murai A, et al. Usefulness of presepsin in the diagnosis of sepsis in a multicenter prospective study. $\mathrm{J}$ Infect Chemother. 2012;18:891-7.

14. Behnes M, Bertsch T, Lepiorz D, Lang S, Trinkmann F, Brueckmann M, et al. Diagnostic and prognostic utility of soluble CD 14 subtype (presepsin) for severe sepsis and septic shock during the first week of intensive care treatment. Crit Care. 2014;18:507.

15. Masson S, Caironi P, Fanizza C, Thomae R, Bernasconi R, Noto A, et al. Circulating presepsin (soluble CD14 subtype) as a marker of host response in patients with severe sepsis or septic shock: data from the multicenter, randomized ALBIOS trial. Intensive Care Med. 2015;41:12-20.

16. Endo S, Takahashi G, Shozushima T, Matsumoto N, Kojika M, Suzuki Y, et al. Usefulnes of presepsin (Soluble CD 14 Subtype) as a diagnostic marker for sepsis. JJAAM. $2012 ; 23: 27-38$.

17. Chenevier-Gobeaux C, Borderie D, Weiss N, Mallet-Coste T, Claessens YE. Presepsin (sCD14-ST), an innate immune response marker in sepsis. Clin Chim Acta. 2015;450:97-103.

18. Arai Y, Mizugishi K, Nonomura K, Naitoh K, Takaori-Kondo A, Yamashita K. Phagocytosis by human monocytes is required for the secretion of presepsin. J Infect 
Chemother. 2015;21:564-9.

19. Urbonas V, Eidukaite A, Tamulienė I. The predictive value of soluble biomarkers (CD14 subtype, interleukin-2 receptor, human leucocyte antigen-G) and procalcitonin in the detection of bacteremia and sepsis in pediatric oncology patients with chemotherapy-induced febrile neutropenia. Cytokine. 2013;62:34-7.

20. Aimoto M, Koh H, Katayama T, Okamura H, Yoshimura T, Koh S, et al. Diagnostic performance of serum high-sensitivity procalcitonin and serum C-reactive protein tests for detecting bacterial infection in febrile neutropenia. Infection. 2014;42:971-9.

21. Masaoka T. Evidence-based recommendations for antimicrobial use in febrile neutropenia in Japan: executive summary. Clin Infect Dis. 2004;39 (Sup 1):S49-52.

22. Tamura K, Imajo K, Akiyama N, Suzuki K, Urabe A, Ohyashiki K, et al. Randomized trial of cefepime monotherapy or cefepime in combination with amikacin as empirical therapy for febrile neutropenia. Clin Infect Dis. 2004;39 (Sup 1):S15-24.

23. From the Immunocompromised Host Society. The design, analysis, and reporting of clinical trials on the empirical antibiotic management of the neutropenic patient. Report of a consensus panel. J Infect Dis 1990; 161: 397-401.

24. Members of the American college of chest physicians/society of critical care medicine consensus conference committee. American College of Chest Physicians/Society of Critical Care Medicine Consensus Conference: definitions for sepsis and organ failure 
and guidelines for the use of innovative therapies in sepsis. Crit Care Med 1992; 20:

864-874.

25. Patel RA, Gallagher JC. Drug fever. Pharmacotherapy 2010; 30: 57-69.

26. Okamura Y, Yokoi H. Development of a point-of-care assay system for measurement of presepsin (sCD14-ST). Clin Chim Acta. 2011;412:2157-61.

27. Chapman P, Reyes C, Gupta V: Normal physiological levels of human cytokines using Bio-Plex Pro ${ }^{\mathrm{TM}}$ cytokine assays. Bio-Plex ${ }^{\circledR}$ suspension array system. [pdf] Available at: < http://www.biorad.com/webroot/web/pdf/lsr/literature/Bulletin_6029.pdf> [Accessed 16 January 2016].

28. Kanda Y. Investigation of the freely available easy-to-use software 'EZR' for medical statistics. Bone Marrow Transplant. 2013;48:452-8. 
Table 1. Baseline characteristics of two prospective cohort populations and details of febrile neutropenia episodes

\begin{tabular}{|c|c|c|}
\hline & Cohort $1(n=43)$ & Cohort $2(n=4)$ \\
\hline Age, years & $45(16-65)$ & $46(29-54)$ \\
\hline Male sex & $19(44.2)$ & $1(25.0)$ \\
\hline \multicolumn{3}{|l|}{ Primary disease } \\
\hline Acute leukemia & $24(55.8)$ & $3(75.0)$ \\
\hline Myelodysplastic syndrome & $4(9.3)$ & $0(0)$ \\
\hline Malignant lymphoma & $7(16.3)$ & $1(25.0)$ \\
\hline Others* & $8(18.6)$ & $0(0)$ \\
\hline \multicolumn{3}{|l|}{ Treatment } \\
\hline Chemotherapy & $52(69.3)$ & $4(100)$ \\
\hline Allogeneic hematopoietic stem cell transplantation & $18(24.0)$ & $0(0)$ \\
\hline Autologous hematopoietic stem cell transplantation & $1(1.3)$ & $0(0)$ \\
\hline None & $4(5.3)$ & $0(0)$ \\
\hline Febrile neutropenic episode, $\mathrm{n}$ & 75 & 4 \\
\hline \multicolumn{3}{|l|}{ Sample collection $\uparrow$} \\
\hline Control sample & 12 & - \\
\hline At onset of febrile neutropenia & 24 & - \\
\hline More than 0 to less than 72 hours after onset of febrile neutropenia & $75(100)$ & - \\
\hline More than 0 to less than 24 hours & $31(41.3)$ & - \\
\hline From 24 to less than 48 hours & $34(45.3)$ & - \\
\hline From 48 to less than 72 hours & $10(13.3)$ & - \\
\hline Neutrophil count at enrollment $(/ \mu \mathrm{l})$ & $67(0-962)$ & $0(0-790)$ \\
\hline Serum creatinine at onset of febrile neutropenia (mg/dL) & $0.66(0.35-1.69)$ & $0.61(0.44-1.03)$ \\
\hline Administration of prophylactic antibiotics & $63(84.0)$ & $2(50)$ \\
\hline Administration of G-CSF & $26(34.7)$ & $2(50)$ \\
\hline \multicolumn{3}{|l|}{ Causes of febrile neutropenia } \\
\hline Septic shock & $5(6.7)$ & 0 \\
\hline Bacteremia & $9(12.0)$ & $1(25)$ \\
\hline Local infection & $27(36.0)$ & $1(25)$ \\
\hline Fever of unknown origin & $31(41.3)$ & $1(25)$ \\
\hline
\end{tabular}


Data are shown as median (range) or number (percentage) of patients.

* Aplastic anemia, $\mathrm{n}=3$; chronic myeloid leukemia, $\mathrm{n}=2$; myelofibrosis, $\mathrm{n}=1$; blastic plasmacytoid dendritic cell neoplasm, $\mathrm{n}=1$

$\dagger$ Because sampling points in Cohort 2 were frequent, according to the protocol guide, the details are described in text.

\$ Two were diagnosed as having drug-induced fever, and a viral infection, in Cohort 1, and one was diagnosed as drug-induced fever in Cohort 2. 


\section{Figure legends}

Fig. 1. Distributions of serum levels of presepsin (a) and procalcitonin (PCT) (b) according to sampling time (at afebrile status before chemotherapy (as control), at onset of febrile neutropenia, and from 0 to less than 24,24 to less than 48 , and 48 to less than 72 hours after onset).

Points above the upper limit of the vertical axis are not included in this figure.

${ }^{*} P<0.05,{ }^{*} P<0.01$, and $* * * P<0.001$ (Steel non-parametric multiple comparison)

$\mathrm{NS}=$ not statistically significant

Fig. 1a

Kruskal-Wallis test $P=0.002$

\section{Fig. $1 b$}

Kruskal-Wallis test $P=0.0004$ 
Fig. 2. Distributions of serum levels of presepsin measured within 72 hours after onset of febrile neutropenia (FN) (a) and procalcitonin (PCT) measured within 72 hours after onset of FN (b) in fever of unknown origin (FUO) $(n=31)$, local infection $(n=27)$, bacteremia $(n=9)$, septic shock $(n=5)$ and others $(n=3)$ groups.

The "others" group included two patients diagnosed as drug-induced fever and one with viral infection.

The horizontal line represents the median value, and points above the upper limit of the vertical axis are not included in this figure.

Fig. 2a

The median value of presepsin within 72 hours of onset of FN with FUO, local infection, bacteremia, septic shock and other conditions was 680 (314-1986) pg/ml, 763 (348-2615), 782 (482-1550), 1359 (493-2114), and 738 (387-1402), respectively (Kruskal-Wallis $P=0.65$; Jonckheere-Terpstra $P=0.089$ for trend ("others" group excluded from both analyses)).

\section{Fig. 2b}

The median value of PCT within 72 hours of onset of FN with FUO, local infection, bacteremia, septic shock and other conditions was $0.15(0.05-5.80) \mathrm{ng} / \mathrm{ml}, 0.13(0.03-1.64), 0.21(0.05-4.82)$, $3.58(0.16-6.22), \quad$ and $0.08 \quad(0.07-0.08), \quad$ respectively (Kruskal-Wallis $P=0.02$; Jonckheere-Terpstra $P=0.105$ for trend ("others" group excluded from both analyses)). 
Fig. 3. Scatter plots of serum levels of presepsin and procalcitonin (PCT) within 72 hours of onset of febrile neutropenia according to subgroups.

Each broken line represents a cut-off level for detecting sepsis: $500 \mathrm{pg} / \mathrm{ml}$ for presepsin and 0.50 $\mathrm{ng} / \mathrm{ml}$ for PCT.

Fig. 3a

Scatter plot for the subgroup with local infection $(\mathrm{n}=27)$

Spearman's rank correlation coefficient $=0.613(P=0.0007)$

Fig. 3b

Scatter plot for the subgroup with bacteremia $(\mathrm{n}=9)$

Spearman's rank correlation coefficient $=-0.200(P=0.613)$ 
Fig. 4. Kinetics of plasma levels of presepsin and serum levels of other biomarkers during episodes of febrile neutropenia (FN) in Cohort $2(n=4)$.

P1, patient no.1; $\mathrm{P} 2$, patient no.2; $\mathrm{P}$, patient no.3; P4, patient no.4; $\mathrm{C} 0$, control (before chemotherapy); C1-C3, the nearest three points to the onset of FN; F0, onset of FN; h, hours after onset of FN; d, days after onset of FN. Points after F11d are not included in this figure.

Reference values: $314 \mathrm{pg} / \mathrm{ml}$ for presepsin, $0.05 \mathrm{ng} / \mathrm{ml}$ for PCT, $0.30 \mathrm{mg} / \mathrm{dl}$ for CRP, $9.00 \mathrm{pg} / \mathrm{ml}$ for IL-6 and $116.00 \mathrm{pg} / \mathrm{ml}$ for IL-8

Fig. 4a: Pattern of fever

Fig. 4b: Plasma presepsin

Fig. 4c: Serum procalcitonin (PCT)

Fig. 4d: Serum C-reactive protein (CRP)

Fig. 4e: Serum interleukin-6 (IL-6)

Fig. 4f: Serum interleukin-8 (IL-8). F0 (787.24 pg/ml) is not included in this figure. 
a

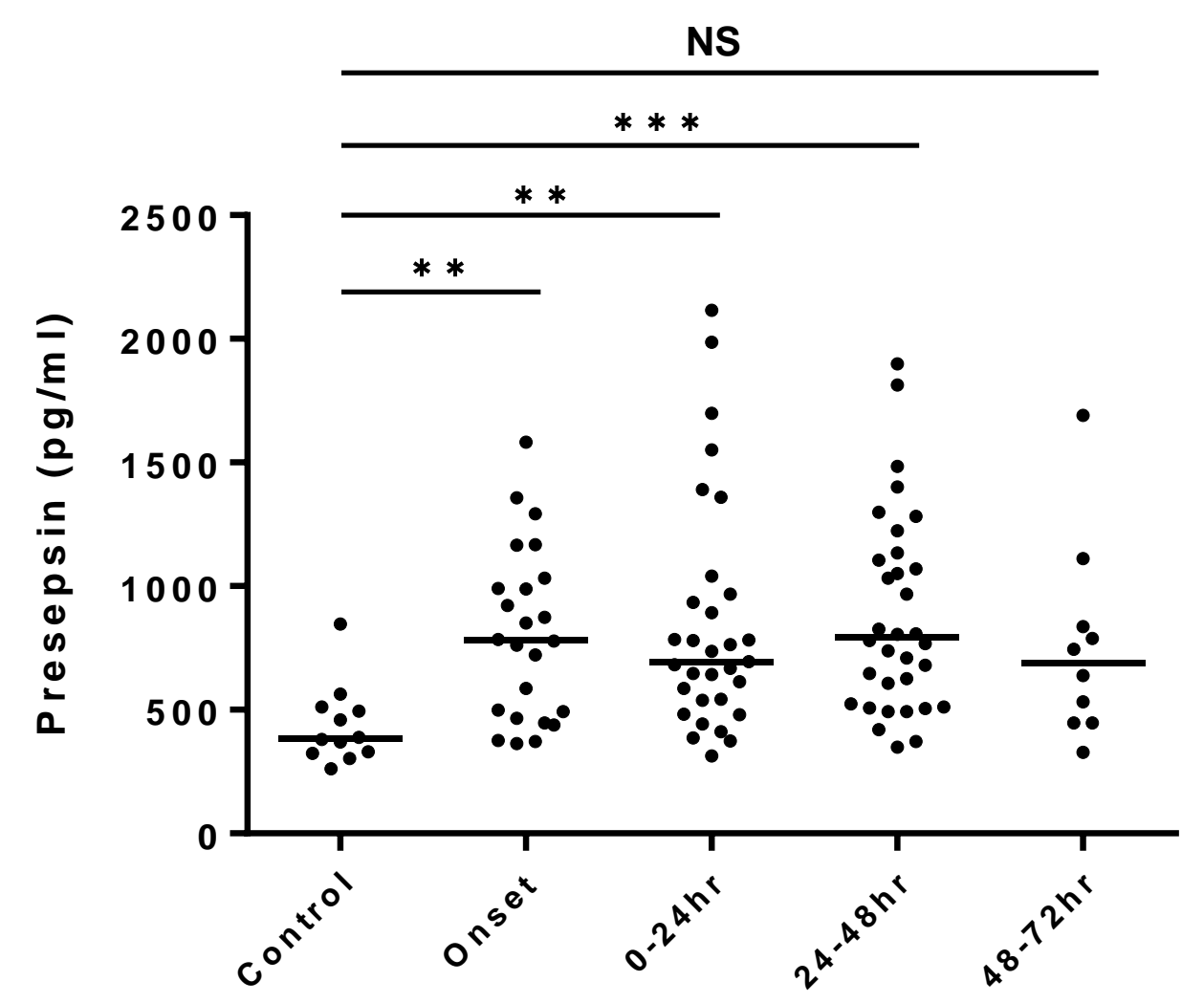

b

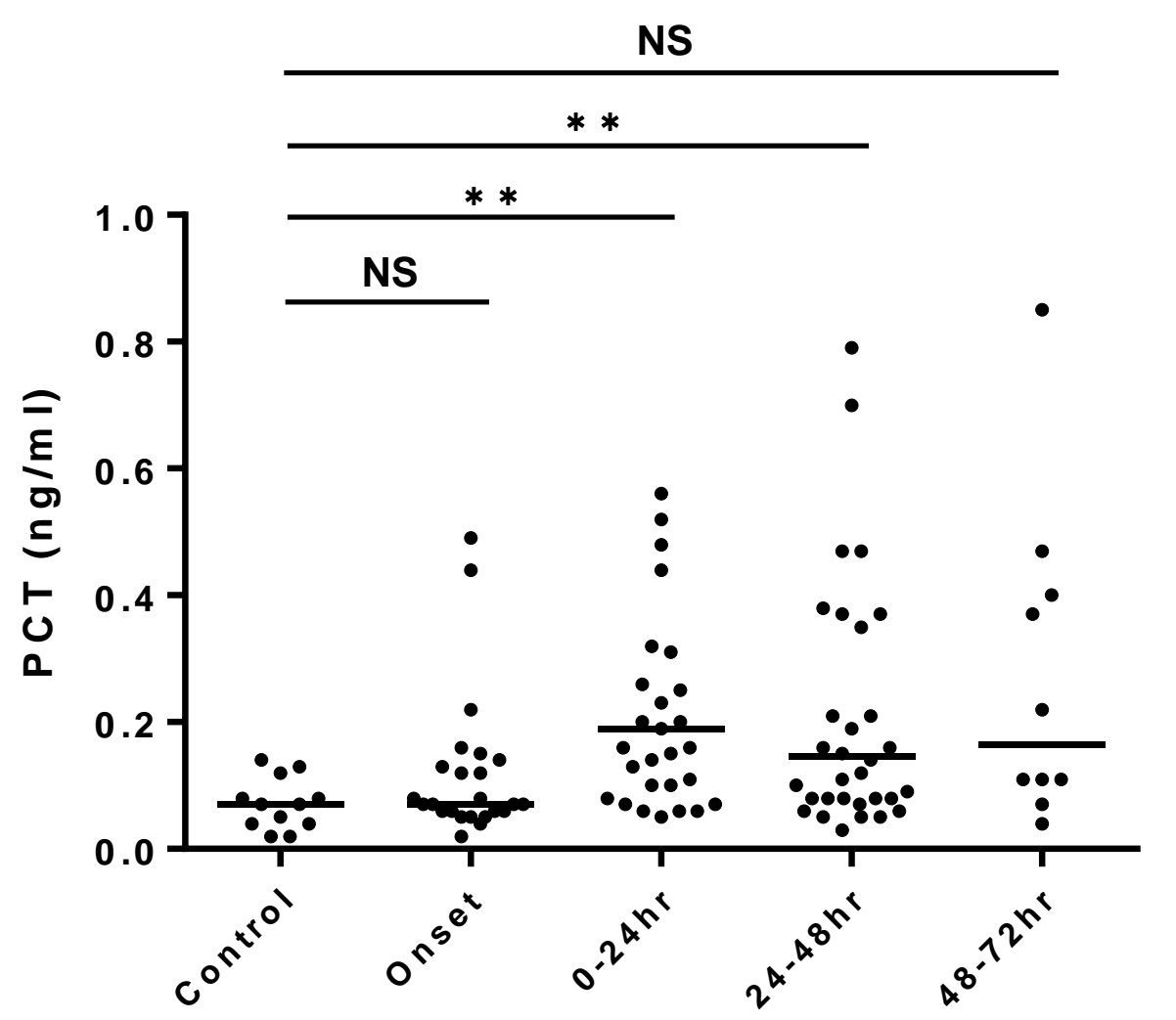



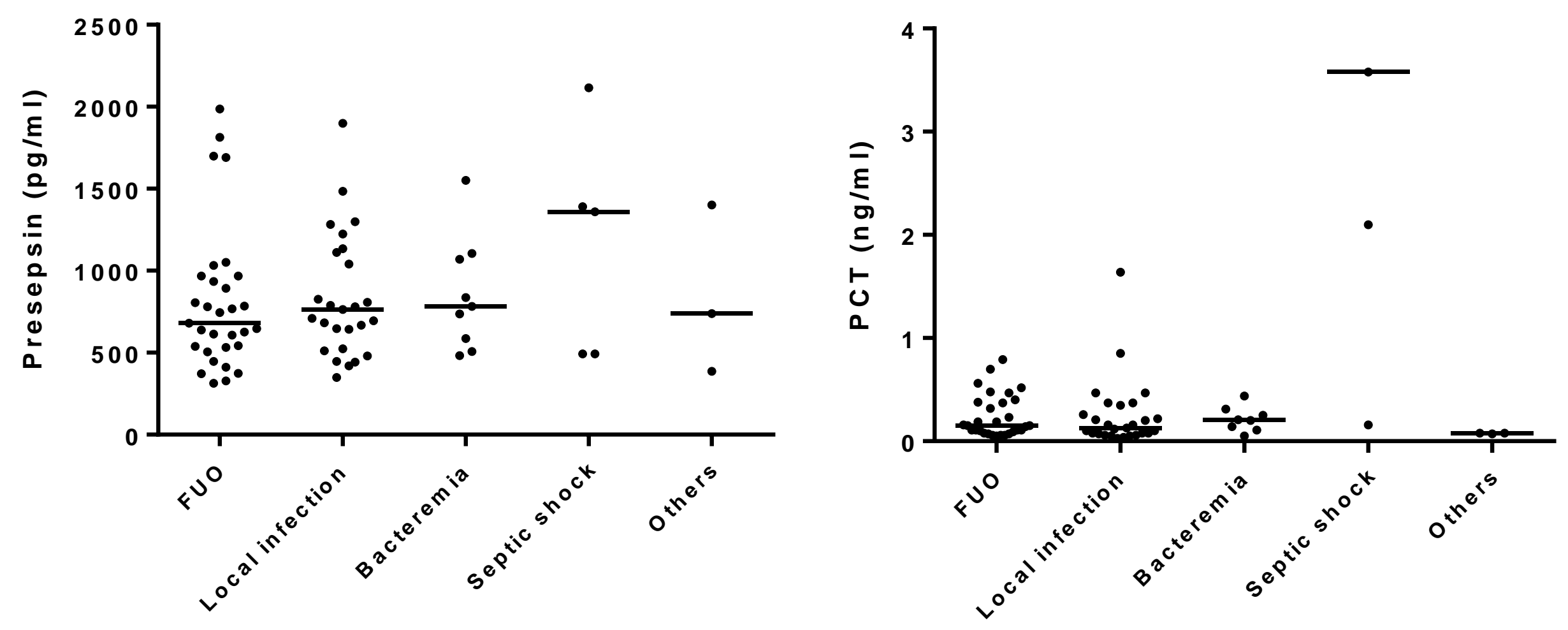

Fig.2 

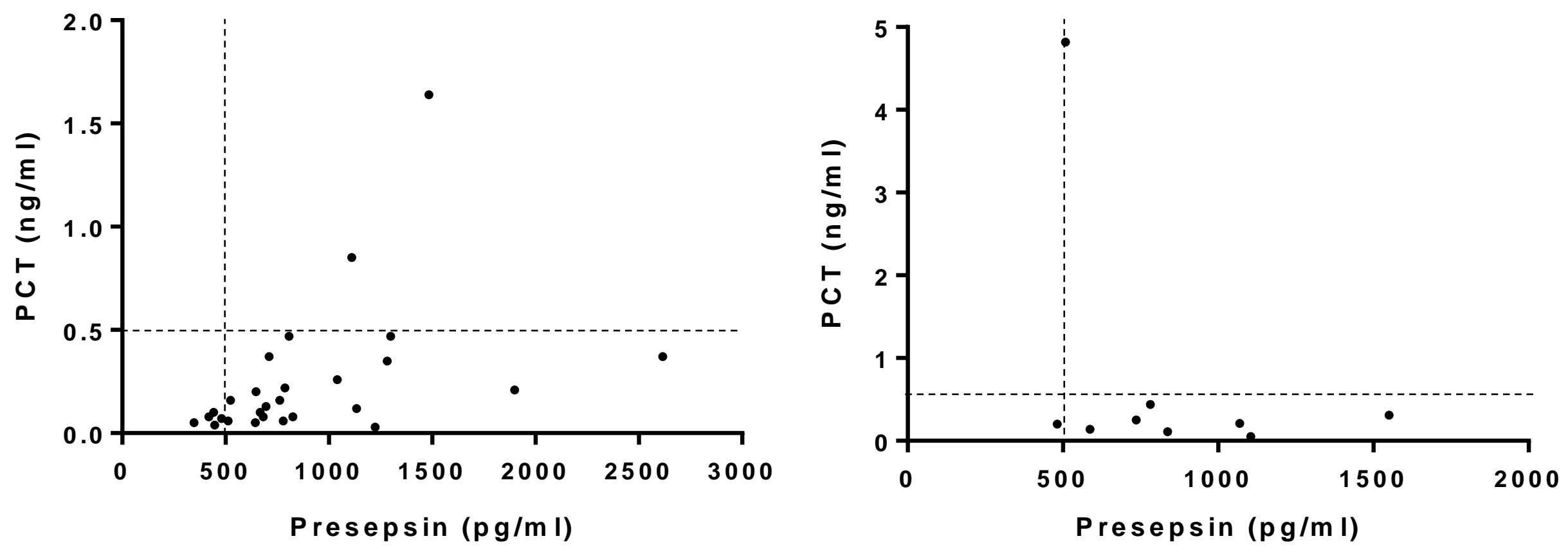
a

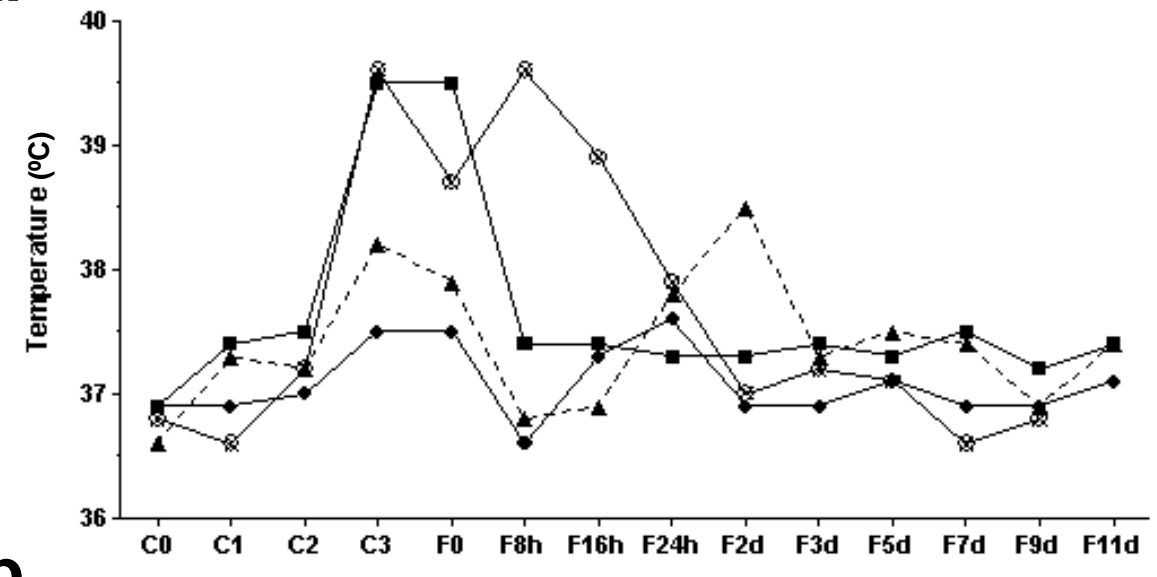

b
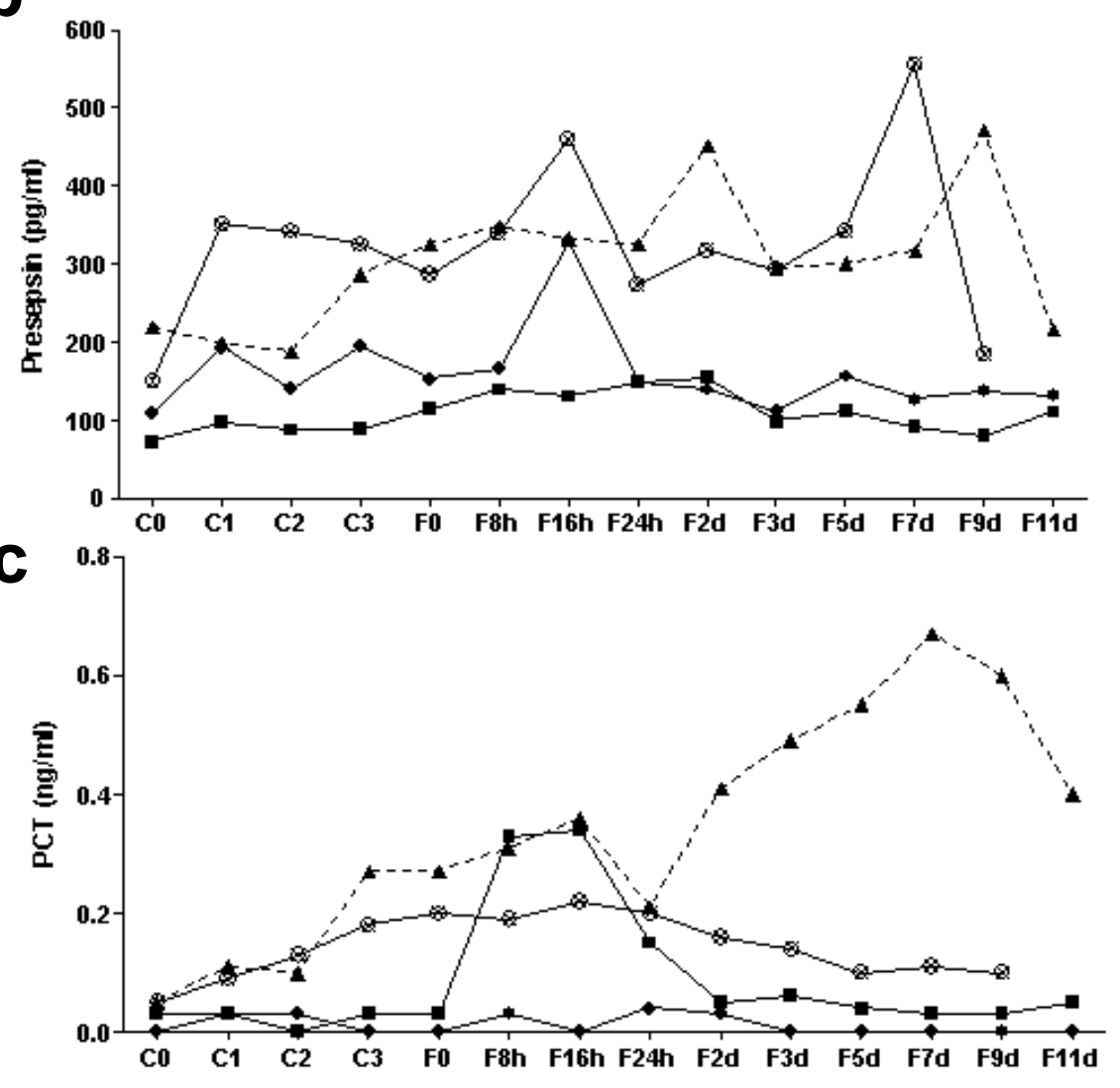

d

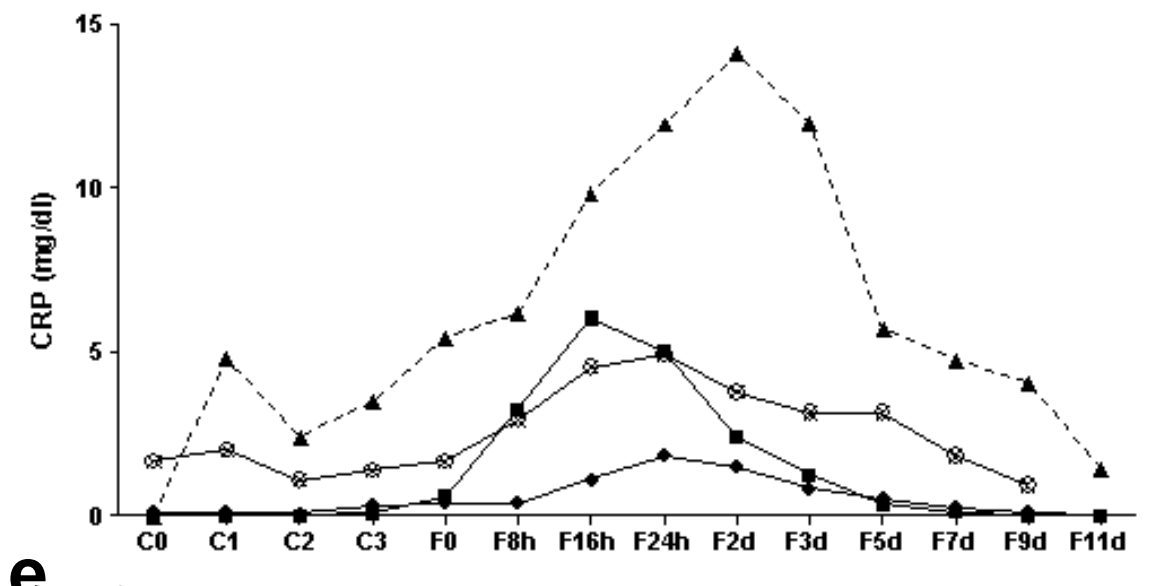

e
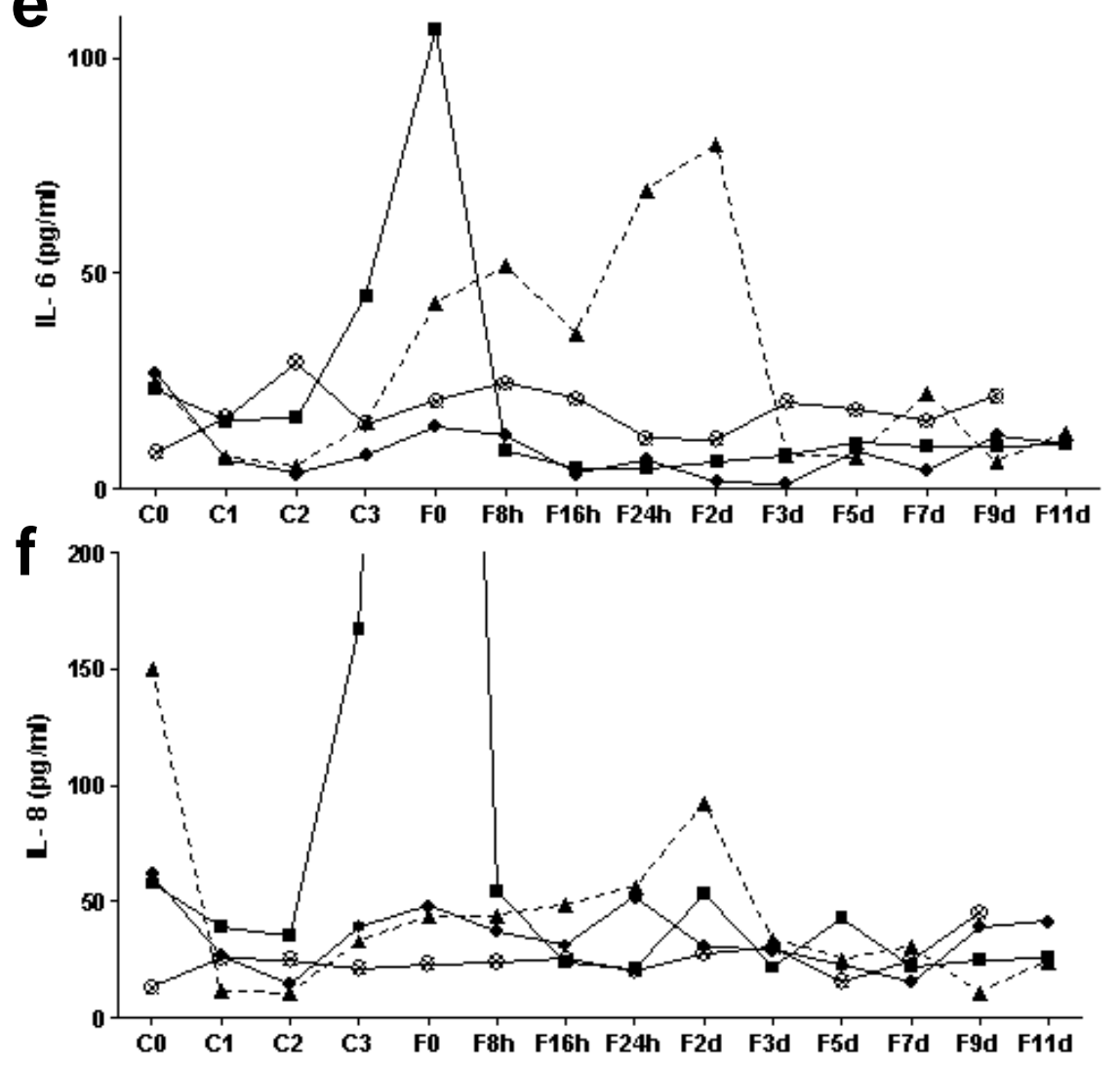

Fig.4 